\title{
Effectiveness of a stress management program in continuous care nursing professionals
}

\section{BACKGROUND}

Nursing is a stressful profession, because it is highly specialized work done under conditions of considerable pressure and has consequently a strong psychological impact. Occupational stress has been considered a serious public health problem, especially in the case of the nursing profession, with considerable consequences for the individual, for the quality of his work and for the associated economic costs. Although the topic of stress has become an important field of research, experimental studies on stress management strategies are rather scarce, especially in the case of the so-called risk professions, such as nursing.

\section{PARTICIPANTS AND PROCEDURE}

The present study applied a cognitive-behavioral training program for stress management to a sample of nursing professionals $(N=5)$ from an intensive care unit. The intervention was applied on the basis of a multiple baseline design. Evaluation of results was carried using quantitative and one qualitative measures. Emphasis was given to evaluation of physiological, cognitive and emotional symptoms of stress.

\section{RESULTS}

The main hypotheses seem to have been confirmed, i.e. the stress management program reduces the participants' physiological, cognitive and emotional stress symptoms according to applied assessment instruments. The results show different levels of stress reduction, as well as an improvement of the number and efficacy of the coping skills of the participants. Correlations were found between quantitative measures, between quantitative and qualitative measures, as well as between physiological, cognitive and emotional symptoms of stress.

\section{CONCLUSIONS}

Research hypotheses were confirmed, i.e., the applied program seems to be able to reduce stress.

\section{KEY WORDS}

occupational stress; stress management; nursing research; cognitive-behavior therapy

ORgANizATION - 1: Comprehensive Health Research Center, University of Evora, Evora, Portugal · 2: Association for

Family Planning, Vilavicosa, Portugal · 3: Research Center in Education and Psychology, University of Evora, Evora, Portugal

AUthors' Contributions - A: Study design - B: Data collection - C: Statistical analysis - D: Data interpretation .

E: Manuscript preparation · F: Literature search · G: Funds collection

Corresponding Author - Prof. Edgar Galindo, Comprehensive Health Research Center, University of Evora,

6 Cardeal Rei Str., 7000 Evora, Portugal, e-mail: edgar_galindo@hotmail.com

to Cite this ARTICLE - Galindo, E., Pires, R., Gracio, L., \& Candeias, A. (2021). Effectiveness of a stress management

program in continuous care nursing professionals. Health Psychology Report, 9(1), 49-62. https://doi.org/10.5114/

hpr.2020.99970

RECEIVED 16.05.2020 • REVIEWED 06.08.2020 • ACCEPTED 26.08.2020 • PUBLISHED 15.10.2020 


\section{BACKGROUND}

Stress has been recently recognized by the World Health Organization (WHO) as "the 21st century health epidemic" because of its devastating effects on the physical and emotional health of human beings (Fink, 2016) and the associated direct and indirect costs. Occupation has been recognized as one of the most important sources of stress for an individual. Hence, occupational stress (OS) is a common, serious and costly problem in the workplace (Saedpanah et al., 2016). Although OS is not specific to a particular professional group, it is thought to be especially prevalent among nursing professionals (Beck, 2011). Several studies carried out over the years have corroborated the idea that nursing is a stressful profession (Hersch et al., 2016; Sarid et al., 2012), due probably to the fact that the nursing career requires sophisticated technical skills, constant vigilance and critical capacity under conditions of considerable pressure and intensity (Zeller \& Lewin, 2013). American studies have shown that there is a clear relationship between the level of staffing by nurses, the proportion of hours of nursing care and better outcomes for hospitalized patients (Needleman et al., 2002). On the other hand, in a statistical survey about nursing career fulfillment in America, it is noted that: "(nurses) are reporting exhaustion a good part of the time or almost always, and 59\% report frustration a good part of the time or almost always" (Reineck \& Furino, 2005, p. 28). Some reviews on the topic of stress in nurses have been published in the past years. For instance, Dunn and Ritter (1995), Edwards and Burnard (2003), Paris and Hoge (2010), and Dickinson and Wright (2008) found, in general terms, that psychological well-being in nurses was related to lower alcohol consumption, being a clinic-based nurse and having full-time work, high self-esteem, good professional fulfillment, management of interprofessional conflicts, involvement in decision making and a reasonable workload. On the other hand, exhaustion was associated with weak professional fulfillment, higher work load, low selfesteem and poor work climate as well as difficulties in nurse relationships, the ability to work together, and staff conflicts. Low involvement in decision making and limited resources. Additionally, American studies point to workload related to an increasingly aged, severely ill, and obese patient population coupled with increasing paperwork. In a study on the relationship between emotional competency and work stress, Humpel and Caputi (2001) found that personnel with more experience had higher levels of emotional competency. Melchior et al. (1997) investigated psychiatric nurses and found that burnout was a result of lower levels of job satisfaction, poor staff support and poor involvement with the organization. In short, there is enough scientific evidence indicating a high risk of chronic stress in this population - a factor associated with the development of burnout syndromes (Bazarko et al., 2013; Zeller \& Lewin, 2013). According to these studies, the most common strategies applied by nurses to cope with stress are recognizing one's own limitations and learning new skills, dealing with problems immediately, keeping good fitness levels, having peer support, and applying personal coping strategies. Additionally, a net of social support, having stable relationships with friends, with the family and partner, a good level of supervision at work, good relations and a set of interests outside of work are important factors to manage stress and maintain good mental health. These known factors seem to have been taken into account to investigate better ways to manage stress. The number of studies devoted to intervention strategies for stress in nurses seems to be smaller than other analyses. Nevertheless, some studies have been made on stress management, including different techniques to reduce stress, improve coping abilities and change the environment to reduce stressors. Milne et al. (1986) applied behavioral techniques to improve therapeutic skills and could improve work satisfaction and levels of sickness as well as reducing stress in psychiatric nurses. Watson (1986) applied relaxation techniques to cope with stress and anxiety. Kunkler and Whittick (1991) used workshops on stress management and progressive muscular relaxation to reduce levels of burnout. Similar results were obtained by Ewers et al. (2002) using psychosocial interventions (training better intervention skills and empathetic work) as a training tool against burnout. However, other forms of intervention did not produce positive results, i.e. social support-based programs to control stress (Carson et al., 1998) and the introduction of innovations in the way of delivering nursing care (Melchior et al., 1996). Behavioral training, relaxation and therapeutic skills taught through workshops for stress management have been applied successfully to stress management (Ewers et al., 2002; Ruotsalainen et al., 2015; Sarid et al., 2012). Interventions based on mindfulness (Gauthier et al., 2015; Moody et al., 2013; Smith, 2014) and meditation (Ando et al., 2011) also claim good results. Nevertheless, the multiple facets and the magnitude of the stress problem, as well as the high contribution of specific social and cultural factors, necessitate further research on scientifically based, effective, simple and economically suitable techniques to manage stress. The development of interventions with the aim of helping nurses to better deal with their work stressors is hence a valid and important objective (Saedpanah et al., 2016; Smith, 2014), especially in countries with a notorious lack of scientifically based intervention studies, like Portugal. The purpose of the present study is to assess the efficacy of a stress management pro- 
gram in a sample of nursing professionals. It is the program "Optimistic Stress Management" developed by Schröder and Reschke (2010) at the University of Leipzig, based mainly on the model proposed by Carson and Kuipers (1998), namely: 1) three levels of the stress process: stressors, moderators of the stress process and stress outcomes; 2) three major sources of external stress: specific occupational stressors, major life events and events produced by 'hassles' (small stressors with a cumulative effect). It is thus a training program based on a cognitive behavioral rationale. The patient with stress problems is trained first to analyze one's own individual stress situations, stressors, reactions, external conditions and symptoms. In the second phase, the patient learns to control the negative cognitions, as well as relaxation and emotional regulation techniques. Emphasis is given to applying effective coping skills, including the development of a social support network. It is usually applied in 10 sessions, but an also recommended short version has been applied in this case. The training program includes assessment instruments, as indicated below.

For the present study, the following hypotheses were defined:

H1: The stress management program reduces the participants physiological, cognitive and emotional stress symptoms according to the Stress Symptoms Questionnaire (SSQ).

$\mathrm{H} 2$ : The stress management program reduces the participants' subjective stress intensity according to the Brief Stress Test (BST).

H3: The program produces a decrease in the participants' stress experience, increasing the number and perceived efficacy of the coping behaviors according to the qualitative measure (QSQ).

H4: All three measures coincide, i.e. the stress measures obtained by BST, SSQ and QSQ coincide.

$\mathrm{H} 5$ : The decreases in the stress levels measured by BST and SSQ occur immediately after the intervention.

H6: Reductions in the stress levels are maintained after training.

\section{PARTICIPANTS AND PROCEDURE}

\section{PARTICIPANTS}

Five participants volunteered for the present study. The socio-demographic characteristics of participants are shown in Table 1.

All sessions took place in a specially assigned room of a convalescent unit of the Portuguese Red Cross, situated in Alentejo region, Portugal. In order to study the efficacy of the intervention, a nonconcurrent interparticipant multiple baseline design was used. A multiple baseline (MBL) design can be used to evaluate intra-subject changes (Johnston \& Pennypacker, 2009). It usually includes more than one participant in a similar context. An initial baseline level is measured for all participants and the dependent variable (in this case the training package) is applied successively to all participants at different moments during the intervention phase (see Table 2). As usual in this kind of study, reliability and validity are granted through experimental control and further replication, instead of a statistical analysis.

After authorization from the University of Evora's ethics committee and the health institution had been granted, the nursing professionals employed by the institution were invited to take part in the study in January, 2017. No selection criteria were applied, because all participants were volunteers. Every participant was delivered an informed consent report with all the information relative to the purpose of the study, data, guarantee of anonymity and compensation for participating. After each participant had signed a declaration of interest, each participant was assigned to a specific intervention time period according to his/her availability. Every participant received a study protocol containing a sociodemographic questionnaire and the assessment instruments BST and SSQ (see later) in order to assess their baseline stress levels. The assessment instrument QSQ (see later) was delivered to each participant in the first and last week of intervention.
Stress in nursing professionals

\section{Table 1}

Socio-demographic characteristics of participants

\begin{tabular}{lccccc}
\hline Participant & Gender & Age & $\begin{array}{c}\text { Marital } \\
\text { situation }\end{array}$ & $\begin{array}{c}\text { Years of professional } \\
\text { experience }\end{array}$ & $\begin{array}{c}\text { Average hours } \\
\text { of work per week }\end{array}$ \\
\hline 1 & Woman & 23 & Single & 1 & 40 \\
2 & Woman & 23 & Single & 2 & 40 \\
3 & Woman & 28 & Married & 3 & 40 \\
4 & Woman & 25 & Single & 4 & 40 \\
5 & Man & 23 & Single & $1 / 2$ & 40 \\
\hline
\end{tabular}


Table 2

Temporal distribution of baseline phase (BL), intervention phase (I) and follow-up (FU) in 5 participants during 22 weeks

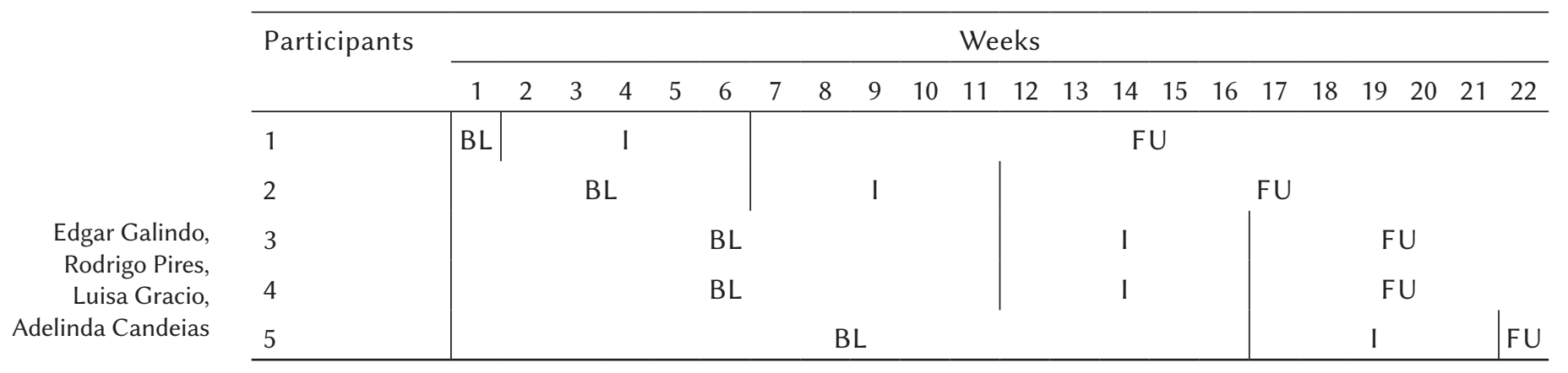

According to the rules of an MBL design, each participant was ascribed to a particular time period in our intervention schedule in order to enroll in the stress management program. Nevertheless, the intervention period always lasted five weeks. Consequently, a baseline level of stress was measured through the above described instruments and then the training package was applied to all participants (see Table 2). A follow-up was carried on after the end of the intervention, with different duration, due to time limitations. After the baseline phase, the first two intervention (I) sessions focused on psychoeducation for stress, different types of stressors and physiological, cognitive, emotional and behavioral stress symptoms. The third I session introduced the rationale for relaxation and started relaxation training. The fourth I session introduced the emotion regulation rationale, training, maladaptive thoughts work and rationale to compensatory leisure activities. The last intervention session focused on personal identity and its relationship with stress and the importance of social support in the various life domains. Additionally, social support maintenance strategies and problem-solving skills applied to stress management were also trained. The stress levels of the participants were evaluated weekly through all phases, with two self-report quantitative assessment instruments as explained below. Additionally, a qualitative questionnaire was applied in the first and in the last training sessions of each participant.

The main instrument is the intervention program "Optimistic Stress Management" (Schröder \& Reschke, 2010) applied in a workshop format during five sessions. This program is a manualized cognitive behavioral intervention focused on stress management consisting of psychoeducation, individual analysis of the stress problem identified by the participant, relaxation training, challenging maladaptive thoughts, emotion regulation training and counselling. In the present study, the intervention was shortened to the five-session format.

\section{MEASURES}

The three following evaluation instruments were used to assess the stress levels of the participants. Two of them (BST and SSQ) are quantitative tests methodized by Schröder and Reschke (2010) and Reschke (2011). The qualitative measure (QSQ) is a questionnaire developed for the present study.

Brief Stress Test (BST; Schröder \& Reschke, 2010). This test assessed the subjective intensity of stress perceived by the participant. This instrument is composed of seven items relative to various aspects of the stress experiences, for example: "Dissatisfaction and anger are my daily companions". The items are associated to one of the following stress themes: loss of control, loss of meaning, anger/dissatisfaction, ability to rest, worrying personal issues, or social support. A 4-point Likert scale has been applied to evaluate the responses of the participants - from 1 (does not apply) to 4 (totally applies). The participant can obtain up to 28 points, denoting the highest level of stress.

Stress Symptoms Questionnaire (SSQ). The SSQ is a 30-item self-report instrument measuring the regularity of the presence of stress symptoms adapted from the instrument introduced by Reschke (2011). The instrument is divided into three scales related to physiological, cognitive and emotional symptoms of stress. Participants are asked to answer to what extent each item corresponds to themselves on a 5-point Likert scale from 0 (little) to 5 (totally). The participant can obtain up to 150 points, denoting the highest level of stress, concerning physiological symptoms (50 points), cognitive symptoms (50 points) and emotional symptoms (50 points). The level of stress of each participant is interpreted according to the level of stress in his/her answers, i.e., 150 points denotes the highest level of stress; consequently, scores between 50 and 100 show a moderate level and less than 50 shows a low level. In the present study a separate observation of the three symptom sets was carried on: (i) bodily symptoms, examples of items: "I have in- 
tense headaches", "I suffer from changes in my heart (palpitations, pain, etc.)"; (ii) cognitive problems, examples: "I have trouble concentrating", "I control myself more and more to avoid mistakes"; (iii) emotional problems, examples of items: "I'm afraid of the future", "I'm out of energy", "I lost my motivation".

Qualitative Stress Questionnaire (QSQ). It was created to assess qualitative reports of participants' stress experience, contextual stressors, usage of coping skills and its perceived efficacy. It is a questionnaire consisting of 6 main questions to assess at the end of the week three main stress dimensions: 1) Intensity of stress felt. Participants answered an open question (Describe the stress felt in the last week - if any), they also mark the rate of stress on a 4-point Likert scale (almost nonexistent, medium, high, very high). 2) Main areas of life where the subject feels stress, sources of stress and its consequences. Participants answered an open-ended question to indicate the stress areas where they felt the most stress (personal life, professional life, health, other aspects); they also indicated on an ordinal scale of two levels the stress felt in each area that they had previously indicated (high, very high) and answer two open response indicators (Aspects that most contribute to my stress; For each of the areas pointed out as the greatest sources of stress, the main consequences for the participant). 3) Subject's proactivity, strategies used to self-regulate the stress felt, self-assessment of their effectiveness and respective causal attribution. The indicators used in this dimension were of different nature: an ordinal scale of two levels to find out if the subjects had done anything to reduce their stress; an open response indicator (What you did to try to decrease the stress felt?); a 4-point Likert scale for self-assessment of the efficacy of strategies to reduce stress (Did what you did to reduce your stress work? Totally, very much, little, it didn't work) followed by an open response indicator (why?).

\section{RESULTS}

\section{RESULTS OF BST: SUBJECTIVE INTENSITY OF STRESS DURING THE PROGRAM}

Figure 1 shows the results obtained by each of the five participants in the weekly application of the Brief Stress Test (BST) during the baseline (BL), the intervention (I) and the follow-up (FU). The number of weeks is shown on the $\mathrm{x}$ axis, and the level of stress obtained by the participant (until 28 scores) on the y axis.

It is evident that participant 1 showed lower stress scores during I and FU (weeks 2-22), compared to BL levels (week 1). It is important to note that the attained low level was maintained during 13 weeks after the end of the training. Participant number 2 showed an increasing level of stress during interven- tion (weeks 7-11), but never exceeding the level of BL. During the FU the level of stress showed an evident constant reduction, attaining a zero level in week 22 , i.e., 11 weeks after the end of training. Participant 3 showed a stable stress level during I (weeks 12-16), compared to BL values, i.e., no change seems apparent. Stress levels show slightly decreasing values during FU (weeks 17-22), remaining stable until the end, at a lower level compared to BL values. Participant 4 showed similar levels of stress during BL (weeks 1-11) and I (weeks 12-16). A decreasing trend of stress level is evident during the FU (weeks 17-22). Participant 5 showed low levels of stress during the BL (weeks 1-16) and an increment during I (weeks 17-21), which is maintained in the only FU measure (week 22). In general terms, it is evident that, independently of the baseline trends, the introduction of training in the intervention phase produces a clear decrease of the stress levels, which is maintained through the follow-up phase. This reduction is maintained until the end of the follow-up, even during many weeks. It is interesting to note that sometimes the decrement is preceded by an increase of stress levels in the intervention phase. The only exception seems to be participant 5, who showed the mentioned increment during training, but not the clear reduction shown by other participants. This is probably due to the fact that the follow-up phase lasted only one week, due to time limitations.

\section{RESULTS OF SSQ: SUBJECTIVE INTENSITY OF STRESS DURING THE PROGRAM}

Figure 2 shows the results obtained by each one of the five participants in the weekly application of the Stress Symptoms Questionnaire (SSQ) during the baseline (BL), the intervention (I) and the follow-up (FU). On the $\mathrm{x}$ axis is shown the number of weeks, and on the $y$ axis the level of stress attained by the participant (up to 150 points) as indicated by all (physiological, cognitive and emotional) symptoms together. Compared to BL, participant 1 showed first an increment of the stress level (weeks 3-4) and then a decrease during I, which was maintained until the end of FU (weeks 7-22). Participant 2 showed an increasing level of stress during BL (weeks 1-6), which diminished steadily during I (weeks 7-11) and during FU (weeks 12-22) attaining almost the level of 0 in the last weeks (21-22). Participant 3 showed a relatively stable level of stress at the end of BL (weeks 1-11) and then a slight reduction during I (weeks 12-16), which remained stable until the end of the FU period, including a level of 0 in week 18. Participant 4 showed low levels of stress during BL (weeks 1-11), a slight increase of stress scores during I (weeks 12-16) and decreasing values attaining 0 during FU. Participant number 5 showed stable levels of stress, with a slight
Stress in nursing professionals 
Figure 1

Results of Brief Stress Test (BST)

Edgar Galindo, Rodrigo Pires, Luisa Gracio, Adelinda Candeias

Participant 1

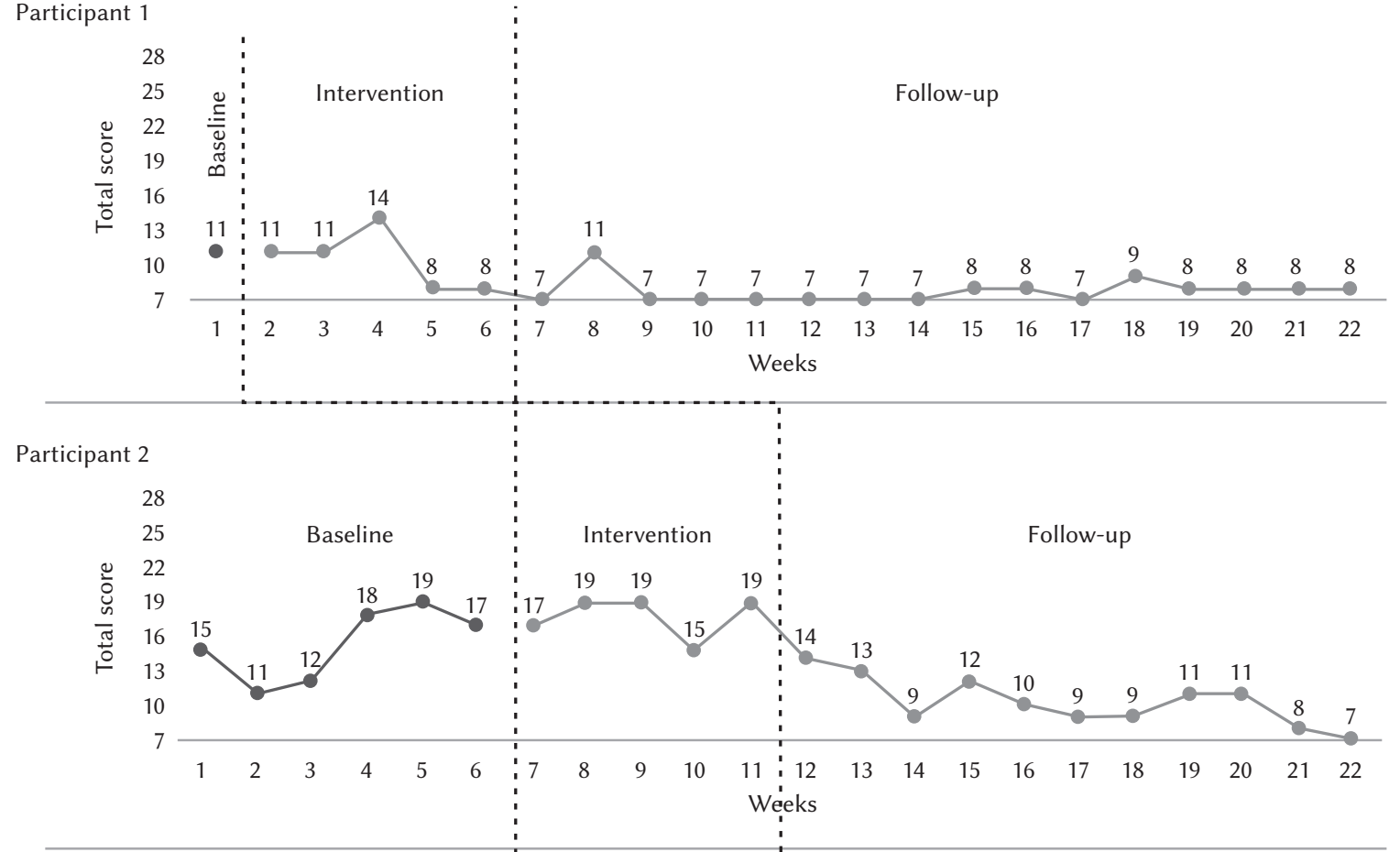

Participant 3

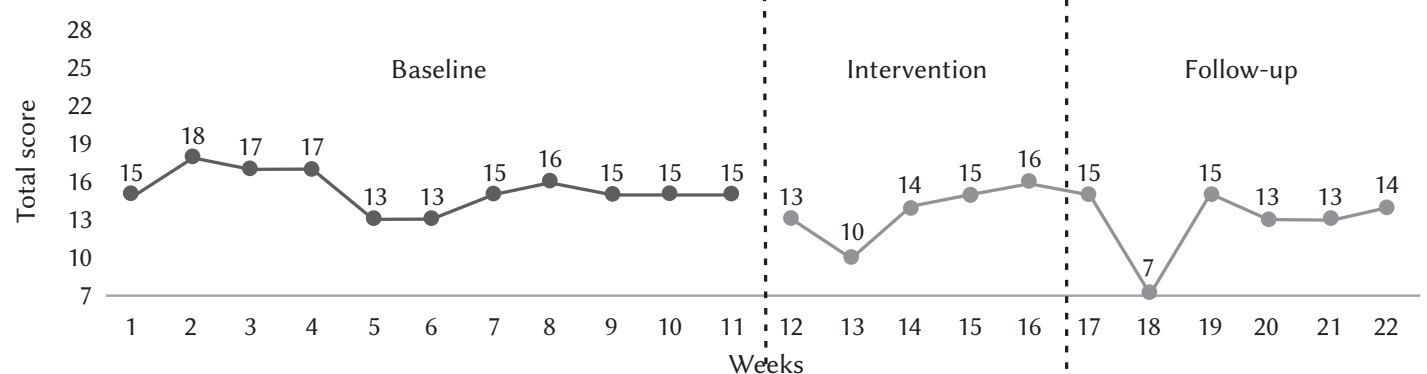

Participant 4

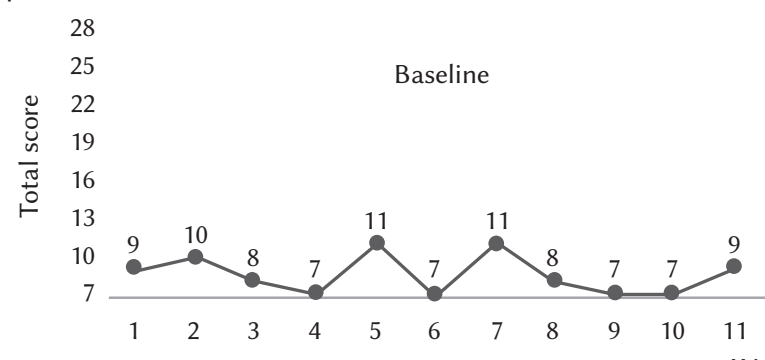

Follow-up

Participant 5

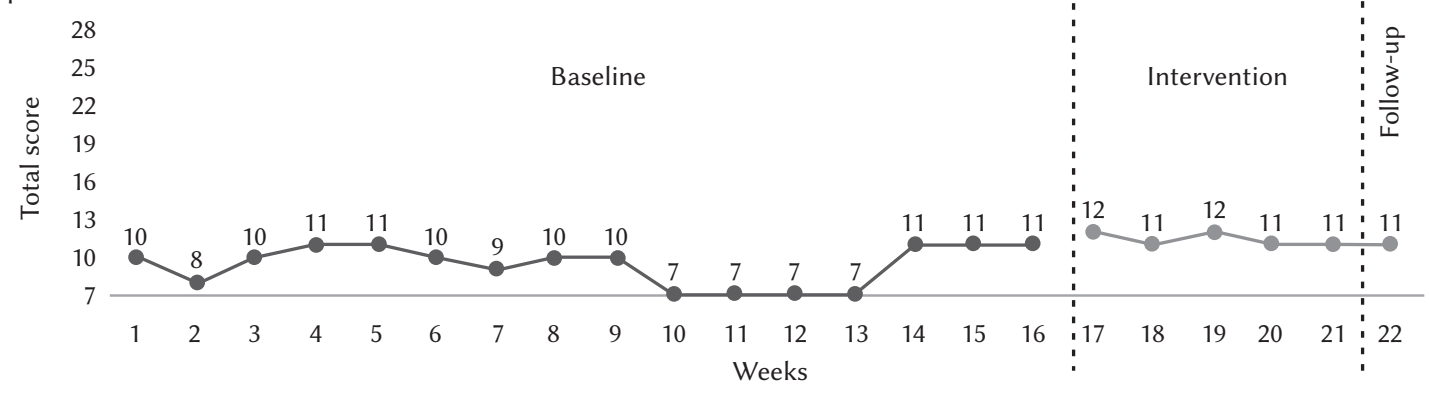


Figure 2

Results of Stress Symptoms Questionnaire (SSQ)

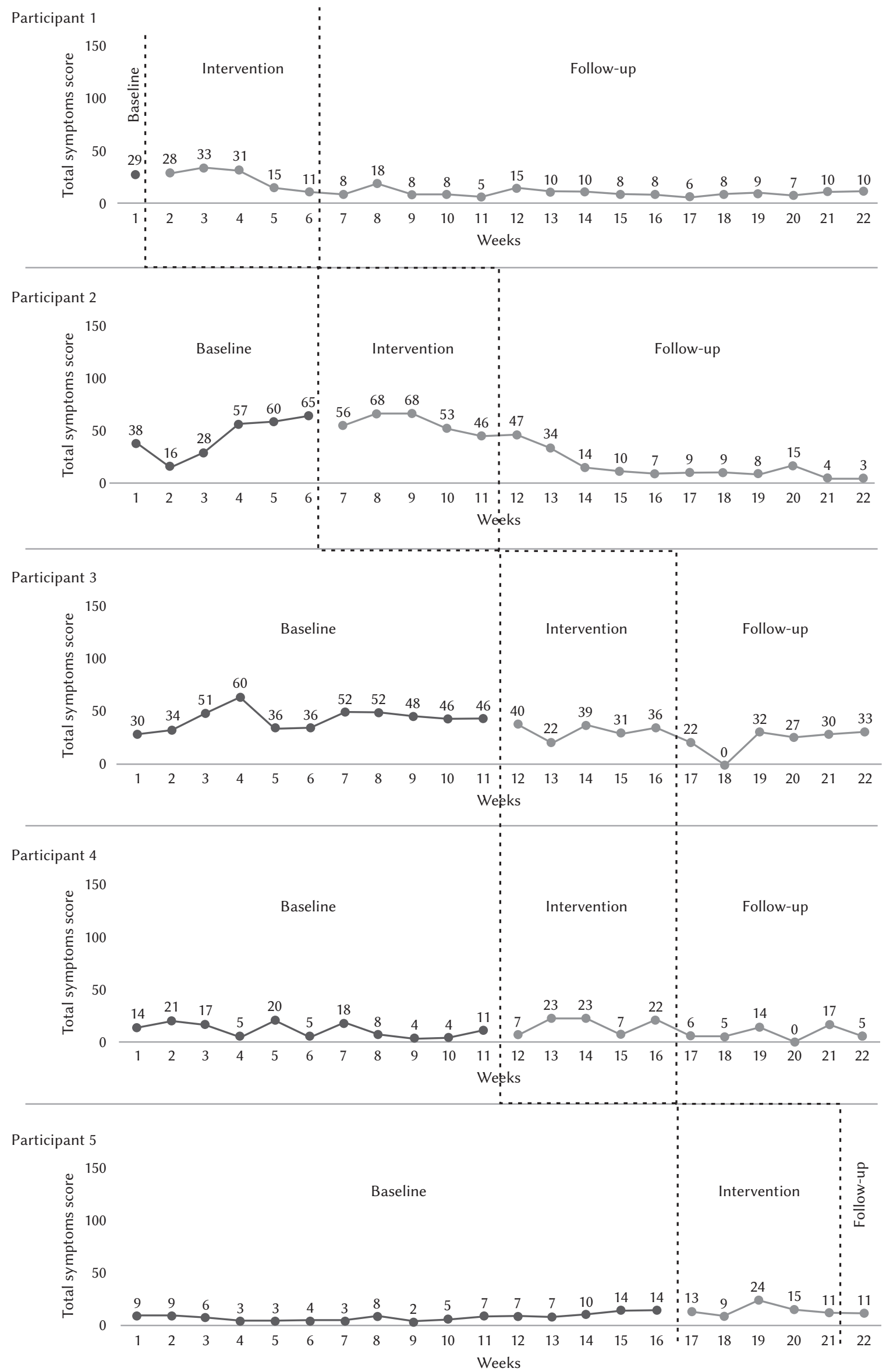

Stress in nursing professionals

articipant 2

Participant

articipant 4

Participant 5 
Edgar Galindo,

Rodrigo Pires, Luisa Gracio, Adelinda Candeias increasing trend during BL (weeks 1-16), a slight increase and a reduction during I (weeks 17-21) and a little reduction at the end of the study, although to levels equal to his baseline scores. In general terms, the results of SSQ show similar trends to the BST results: independently of the baseline trends, the introduction of training in the intervention phase produces a clear decrease of the stress levels, through the follow-up phase, until the end of the study. Participant 5 is the exception.

\section{SSQ PHYSIOLOGICAL SYMPTOMS RESULTS}

Figure 3 shows the results obtained by each one of the five participants in the weekly application of the physiological symptoms scale of SSQ during BL, I and FU. On the $\mathrm{x}$ axis is shown the number of weeks, and on the $y$ axis the level of stress attained by the participant (up to 50 points) as indicated by physiological symptoms. Compared to BL, Participant 1 showed a clear reduction of the physiological stress symptoms from BL (week 1), through I (weeks 2-6) to the end of the FU phase (weeks 7-22). Participant 2 showed similar results, i.e., a clear reduction of physiological stress scores from BL (weeks 1-6) through I (weeks 7-11) to FU (weeks 12-22), attaining almost 0. Similar results were shown by participant 3 , with decreasing levels of physiological symptoms from BL (weeks 1-11), through I (weeks 12-16) until FU (weeks 17-22). Participant 4 showed similar low scores in BL (weeks 1-11), and I (weeks 12-16), but slightly lower scores attaining 0 in FU (weeks 17-22). Participant 5 showed a low stress level in BL (weeks 1-16), an increment until 11 points in I (weeks 17-21) and then a decline in the only FU session (week 22). Nevertheless the stress levels in I and FU were higher than in BL. In general terms, it is evident that participants 1, 2, 3 and 4 showed at the end of the intervention lower levels of physiological symptoms than in BL. Participant 5 is an exception.

\section{SSQ COGNITIVE SYMPTOMS RESULTS}

Figure 4 shows the results obtained by each of the five participants in the weekly application of the cognitive symptoms scale of SSQ during BL, I and FU. On the $\mathrm{x}$ axis is shown the number of weeks, and on the $y$ axis the level of stress attained by the participant (up to 50 points) as indicated by cognitive symptoms. Participant 1 showed a steady reduction of cognitive stress symptoms during I (weeks 2-6) in comparison to BL (week 1). This reduction was maintained until the end of the FU phase (weeks 7-22). Participant 2 showed an increase of the cognitive stress symptoms during $\mathrm{BL}$ (weeks 1-6), this trend changing to start decreasing with the onset of I (weeks 7-11) through FU, attain- ing a level of 0 by the end of the phase. Participant 3 showed a relatively stable level of cognitive stress symptoms during BL (weeks 1-11), and then a slight decrease during I. In the FU phase (weeks 17-22), a stress level lower than BL was maintained, attaining a level of 0 in one week. Participant 4 showed a very low level of cognitive stress symptoms, including four weeks with 0 scores, during BL (weeks 1-11) and then a slight increase during I (weeks 12-16)., but always lower than BL values. These low stress values were maintained during FU (17-22), including four 0 level measures. Participant 5 showed a very low level of cognitive stress symptoms through $16 \mathrm{BL}$ weeks. A slight stress increased occurred during I (weeks 17-21), and the last measure during FU showed a final level of stress of 1 point. In general terms, it is evident that the results obtained by participants in terms of physiological and cognitive symptoms correlate with ups and downs. On the other hand, all participants, including this time participant 5 , showed at the end of the intervention lower levels of cognitive symptoms than in BL.

\section{SSQ EMOTIONAL SYMPTOMS RESULTS}

Figure 5 shows the results obtained by each of the five participants in the weekly application of the emotional symptoms scale of SSQ during BL, I and FU. On the $\mathrm{x}$ axis is shown the number of weeks, and on the $\mathrm{y}$ axis the level of stress attained by the participant (up to 50 points) as indicated by emotional symptoms. Participant 1 showed an increase of emotional stress symptoms during I (weeks 2-6) in comparison to BL (week 1). A constant reduction of the stress level is noticeable in the FU phase (weeks 7-22), attaining 2 points in the last two weeks. Participant 2 showed a clear increment of the emotional stress scores during I (weeks 7-11) compared to BL levels (weeks 1-6). A decrease began by the end of I, which continued steadily during FU (weeks 12-22), attaining the lowest level in the last two weeks. Participant 3 showed a relatively high stress level as indicated by emotional symptoms during BL (weeks 1-11), with a reduction by the onset of I (weeks 12-16), which was maintained through the FU phase (weeks 17-22), including one week with a 0 level of stress. Participant 4 showed variable levels of emotional stress during BL (weeks 1-11), a clear increment during I (weeks 12-16) and then a clear decrease of the emotional stress symptoms during FU (weeks 17-22), including three weeks with a 0 stress level. It is evident that participants 1, 2, 3 and 4 showed at the end of the intervention lower levels of emotional symptoms than in BL. Participant 5 is an exception. In general terms, it is evident that the results obtained by participants in terms of physiological, cognitive and emotional symptoms correlate with ups and downs. On the other hand, all 
Figure 3

Psychological symptoms score. Results of the psychological symptom scale of Stress Symptoms Questionnaire (SSQ)
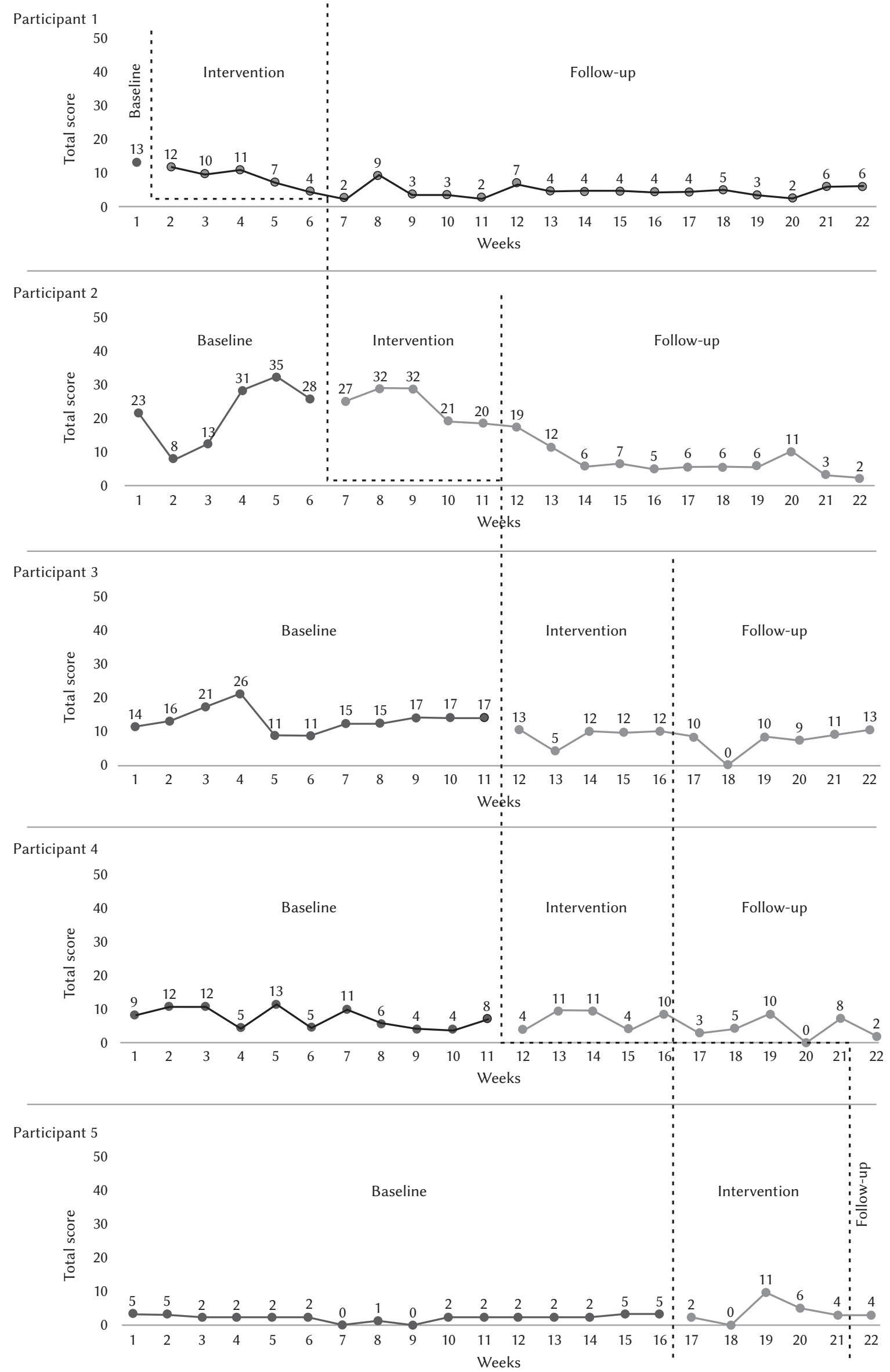


\section{Figure 4}

Cognitive symptoms score. Results of the cognitive symptom scale of Stress Symptoms Questionnaire (SSQ) Participant 1

Edgar Galindo, Rodrigo Pires, Luisa Gracio, Adelinda Candeias

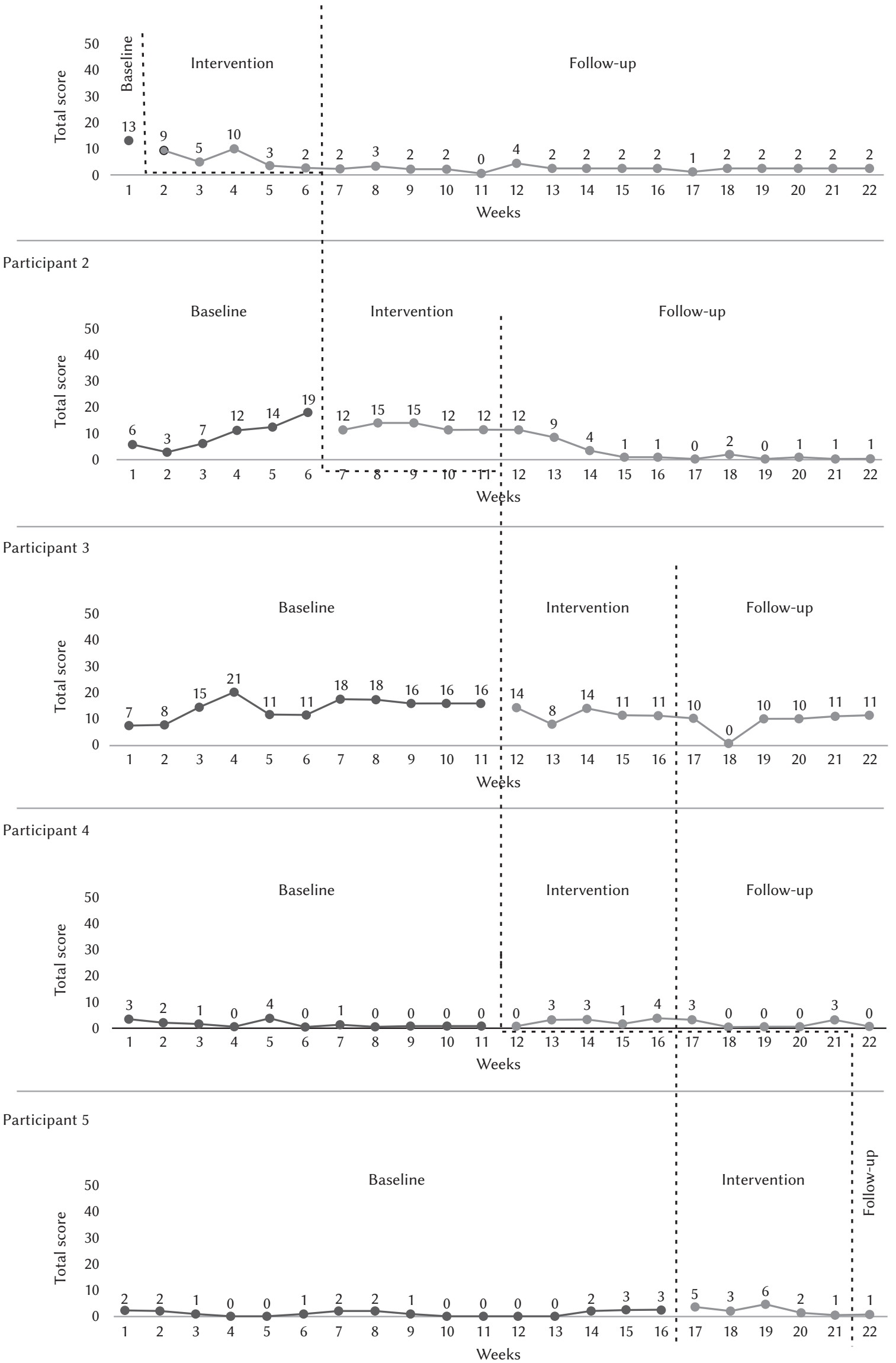


Figure 5

Emotional symptoms score. Results of the emotional symptom scale of Stress Symptoms Questionnaire (SSQ) Participant

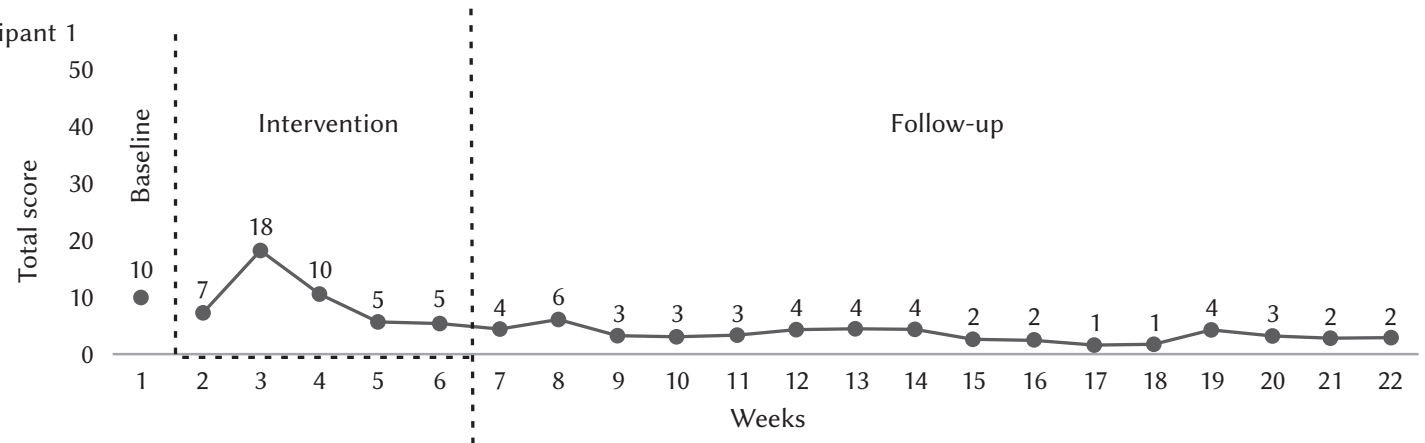

Stress in nursing professionals
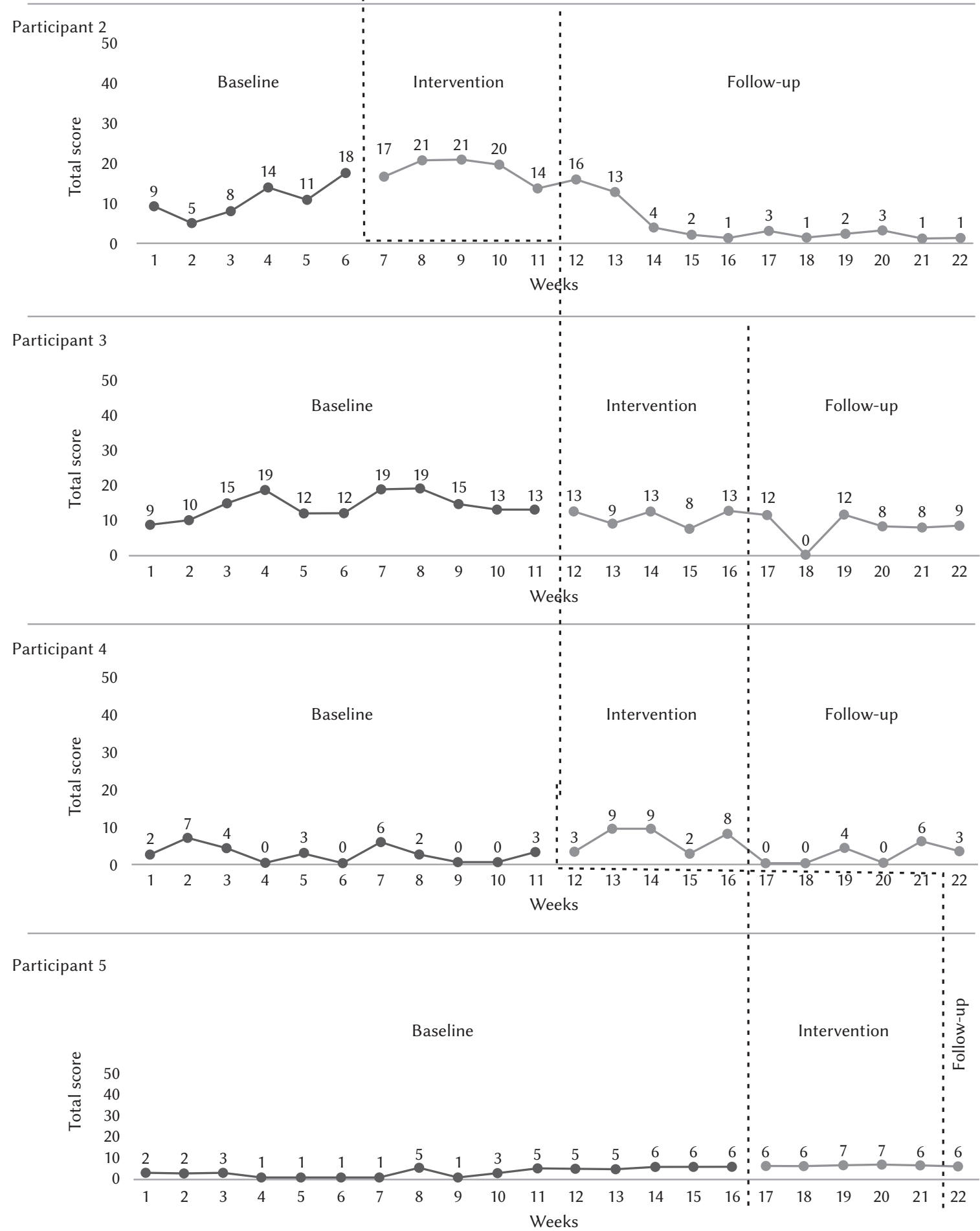
participants showed at the end of the intervention lower levels of cognitive symptoms than in BL. The exception is participant 5. Concerning the measurement of physiological, cognitive and emotional symptoms, correlations are evident. Nevertheless, some variations were interesting. For instance, participant 3 showed clear steady reductions in cognitive and emotional scores, which were not accompanied by clear reductions in physiological scores.

Edgar Galindo, Rodrigo Pires, Luisa Gracio, Adelinda Candeias

\section{QSQ: SUBJECTIVE INTENSITY OF STRESS DURING THE PROGRAM. PRE-INTERVENTION RESULTS}

Prior to the intervention phase, the QSQ showed that the type of stressors to which the participants were exposed in the previous week were related to "professional life" and "personal life". All reports inside these two categories were rated as medium intensity. Participant number 1 was the only one who did not respond to this question, although rating it as mainly inexistent. As for the major life areas which were sources of stress, three different areas were highlighted by the participants: "personal life", "professional life" and "health". Every major life area referred to by the participants was rated high in intensity. The consequences of stress exposure reported by the participants were of three types: "emotional", "cognitive" and "physiological". The stress reduction techniques employed by the participants involved "leisure activities" and "breathing control". Only participant 2 did not use them.

\section{QSQ POST-INTERVENTION RESULTS}

Regarding the stressors present in the week prior to the application of the post-intervention questionnaire, the participants' responses refer to three different aspects: "professional life", "personal life" and "individualized aspects". One of them reported the absence of stressors and another one did not answer. In professional life the aspects related to stress in the last week are "unexpected events" and "work overload". Regarding "personal life" the reported stress consisted in "homebuilding problems". In the nonexistent category, participant 4 reported: "Last week I felt less stress. I had good news that the new house project was approved. There have been easier days". As for the intensity of the stressors experienced the previous week, participants 1, 4 and 5 rated their intensity as almost nonexistent and participants 2 and 3 as average. As for the major life areas which were source of stress, it should be noted that two of the pre-test categories have been maintained: "personal life" and "professional life". In professional life, the stress reported by the participants refers to "work overload". All participants reported a high degree of stress in the above-mentioned life areas. As for the consequences of stress experienced for the participants, the categories obtained during the pretest were maintained: "emotional", "cognitive" and "physiological". Within the emotional consequences, the experienced stress is related to irritability and anxiety and in the cognitive consequences, the stress experienced is expressed in the form of negative thinking and successive thoughts. Finally, physiological consequences of the stress experienced by the participants are expressed through fatigue, tension, sweating, sleep disturbance and tachycardia. All participants stated that they used techniques to reduce stress. Hence, participants' responses are relative to activities such as "leisure", "sports activity", "seeking social support", "rest", "breathing control" and "problem solving". Lastly, the perceived efficacy of the stress reduction strategies used was reported by all participants as very high for the following reasons: "Because I can perceive the physical and mental changes that help me work better" (participant 1); "While I was at the cafe, I was not thinking about things that were stressing me, and when I spoke with others, I felt relieved and settled the matter" (participant 2); "Because I can restore energies with rest and abstract and socialize when I do physical activity in the gym" (participant 3); "I had fewer doubts, decreased fears and I felt less anxious" (participant 4); "Because it allows my stress levels to decrease [...] It gets the job done" (participant 5).

\section{DISCUSSION}

Results from our quantitative measures of stress (BST and SSQ) show an evident stress reduction in the participants of the present study. They show also that physiological, cognitive and emotional measures of stress according to SSQ correlate evidently. Stress reductions are maintained until several weeks after the end the intervention, according to follow-up observations. It is also evident that all positive changes occur after the introduction of training and never before. These data show that "Optimistic Stress Management" seems to be a good help to reduce stress experience. Of course, a word must be said about participant 5 . In this case, gains are not evident. It may be due to the fact that the follow-up period was too short, or it might be that the levels of stress of the participant were too low from the very beginning, but the question is open to discussion and further research. In any case, the multiple baseline design has been demonstrated to be a useful tool to analyze small personal differences, a fact of major interest in this kind of research. Back to the participants, although gains seem to be small, it must be taken into account that a decrease in only one symptom may represent a big relief 
for the person. Qualitative results can help to better definition of the real extent of the therapeutic gains. First, although there was an increase in the total number of stressors identified, an increase in the total verbalizations relative to consequences of stress experienced by the participants and that the major areas perceived as source of stress remained unaltered after the intervention, it does not imply that participants had more stress following the intervention. Rather, the fact that participants 1,2, 4 and 5 showed some increases in the total stress symptoms as measured by the SSQ during the intervention may mean that participants are more capable of identifying and recognizing the stressors present in their life contexts, on one hand, and their stress reactions on the other. This could be due to the psychoeducation received in the intervention phase, which focuses on these topics. Another important aspect of our qualitative data concerns the stress reduction techniques reported by the participants. Before the intervention, one of the participants reported that she did not engage in any stress reduction type activity, and two of the remaining four participants perceived the efficacy of their stress reduction techniques as little. After the intervention, we can see that not only every participant reported engaging in stress reduction techniques, but also that the total number of techniques reported by the participants doubled from three to six different types. Also, the new strategies reported by the participants are congruent with aspects covered in the intervention program, namely problem-solving skills, resting and social support. It is also important to note that there was a general increase in the perceived efficacy of the stress reduction techniques reported by the participants, since all of the participants rated their perceived efficacy as very high. The qualitative results also seem to point not only to the development of new coping skills, but also to the improvement of existing skills prior to the intervention. For example, participant 5, before the intervention, reported using "breath control" to reduce his stress but rated his perceived efficacy in doing so as a little because "[I] think too much about my problems". After the intervention, participant 5 reported that he dealt with his stress through a combination of "breathing control" and "problem solving", perceived by the subject as very efficacious, arguing that "[...] it allows my stress levels to decrease [...] It gets the job done". According to Folkman (2013) this seems to indicate the use of coping skills used in conjunction, which can also explain the increase in the perceived efficacy.

\section{CONCLUSIONS}

All research hypotheses were confirmed, which means that the "Optimistic Stress Management" program seems to be able to reduce stress experience, to promote a better capacity to recognize stressors and stress reactions, as well as to promote new coping skills and/or improve previous coping skills prior to the intervention. On the other hand, quantitative and qualitative measures of stress, as well as measures of cognitive, physiological and emotional symptoms of stress, correlated. It is important to emphasize that the personal reports of participants at the end of the study confirm the application of anti-stress strategies learned during intervention. Last but not least, it was demonstrated that the multiple baseline design is able to give valuable information in this kind of research. These findings can open new areas of research and help to develop better intervention techniques for stress management. Future studies will need to replicate the present study with other nursing professionals from the same setting but also from other occupational settings in order to fully comprehend the extent to which the results may be verified. Some limitations of the current study are as follows: 1) the assessment instruments could be more reliable, a biological measurement of stress levels would increase the reliability of the study; 2) in this kind of study, replication is an essential step of scientific demonstration, i.e., replications with other populations and in other contexts; 3 ) the participants are relatively young people with little experience and this may affect the baseline stress level and the effectiveness of the intervention.

\section{RefERENCES}

Ando, M., Natsume, T., Kukihara, H., Shibata, H., \& Ito, S. (2011). Efficacy of mindfulness-based meditation therapy on the sense of coherence and mental health of nurses. Health, 3, 118-122. https:// doi.org/10.4236/health.2011.32022

Bazarko, D., Cate, R. A., Azocar, F., \& Kreitzer, M. J. (2013). The impact of an innovative mindfulnessbased stress reduction program on the health and well-being of nurses employed in a corporate setting. Journal of Workplace Behavioral Health, 28, 107133. https://doi.org/10. 1080/15555240.2013.779518

Beck, C. T. (2011). Secondary traumatic stress in nurses: a systematic review. Archives of Psychiatric Nursing, 25, 1-10. https://doi.org/10.1016/j. apnu.2010.05.005

Carson, J., Butterworth, T., \& Burnard, P. (1998). Clinical supervision, stress management and social support. In T. Butterworth, J. Faugier, \& P. Burnard (Eds.), Clinical Supervision and Mentorship in Nursing (pp. 49-65). Stanley Thornes.

Carson, J., \& Kuipers, E. (1998). Stress management interventions. In S. Hardy, J. Carson, \& B. Thomas (Eds.), Occupational stress: Personal and professional approaches (pp. 157-174). Stanley Thornes.

Dickinson, T., \& Wright, K. M. (2008) Stress and burnout in forensic mental health nursing: a liter-
Stress in nursing professionals 
Edgar Galindo,

Rodrigo Pires, Luisa Gracio, Adelinda Candeias ature review. British Journal of Nursing, 17, 81-87. https://doi.org/10.12968/bjon.2008.17.2.28133

Dunn, L., \& Ritter, S. (1995). Stress in mental health nursing. A review of the literature. In J. Carson, L. Fagin, \& S. Ritter (Eds.), Stress and coping in mental health nursing (pp. 29-45). Chapman \& Hall.

Edwards, D., \& Burnard, P. (2003). A systematic review of stress and stress management interventions for mental health nurses. Journal of Advanced Nursing, 42, 169-200. https://doi.org/10.1046/j.13652648.2003.02600.x

Ewers, P., Bradshaw, T., McGovern, J., \& Ewers, B. (2002). Does training in psychosocial interventions reduce burnout rates in forensic nurses? Journal of Advanced Nursing, 37, 470-476. https:// doi.org/10.1046/j.1365-2648.2002.02115.x

Fink, G. (2016). Stress, definitions, mechanisms, and effects outlined: Lessons from anxiety. In G. Fink (Ed.), Stress: Concepts, cognition, emotion, and behavior (pp. 33-37). Elsevier.

Folkman, S. (2013). Stress: Appraisal and coping. In M. D. Gellman \& J. R. Turner (Eds.), Encyclopedia of behavioral medicine (pp. 1913-1915). Springer.

Gauthier, T., Meyer, R. M., Grefe, D., \& Gold, J. I. (2015). An on-the-job mindfulness-based intervention for pediatric ICU nurses: a pilot. Journal of Pediatric Nursing, 30, 402-409. https://doi.org/10.1016/j. pedn.2014.10.005

Hersch, R. K., Cook, R. F., Deitz, D. K., Kaplan, S., Hughes, D., Friesen, M. A., \& Vezina, M. (2016). Reducing nurses' stress: a randomized controlled trial of a web-based stress management program for nurses. Applied Nursing Research, 32, 18-25. https:// doi.org/10.1016/j.apnr.2016.04.003

Humpel, N., \& Caputi, P. (2001). Exploring the relationship between work stress, years of experience and emotional competency using a sample of Australian mental health nurses. Journal of Psychiatric and Mental Health Nursing, 8, 399-403. https://doi. org/10.1046/j.1365-2850.2001.00409.x

Johnston, J. M., \& Pennypacker, H. S. (2009). Strategies and tactics of behavioral research. Routledge.

Kunkler, J., \& Whittick, J. (1991). Stress-management groups for nurses: Practical problems and possible solutions. Journal of Advanced Nursing, 16, 172-176. https://doi.org/10.1111/j.1365-2648.1991. tb01621.x

Melchior, M., Bours, G., Schmitz, P., \& Wittich, Y. (1997). Burnout in psychiatric nursing: a metaanalysis of related variables. Journal of Psychiatric and Mental Health Nursing, 4, 193-201. https://doi. org/10.1046/j.1365-2850.1997.00057.x

Melchior, M., Philipsen, H., Abu-Saad, H., Halfens, R., van de Berg, A., \& Gassman, P. (1996). The effectiveness of primary nursing on burnout among psychiatric nurses in long-stay settings. Journal of Advanced Nursing, 24, 694-702. https://doi. org/10.1046/j.1365-2648.1996.02457.x
Milne, D., Burdett, C., \& Beckett, J. (1986). Assessing and reducing the stress and strain of psychiatric nursing. Nursing Times, 82, 59-62.

Moody, K., Kramer, D., Santizo, R. O., Magro, L., Wyshogrod, D., Ambrosio, J., Castillo, C., Lieberman, R., \& Stein, J. (2013). Helping the helpers: mindfulness training for burnout in pediatric oncology, a pilot program. Journal of Pediatric Oncology Nursing, 30, 275-284. https://doi.org/10.1177/ 1043454213504497

Needleman J., Buerhaus, P., Mattke, S., Stewart, M., \& Zelevinsky, K. (2002). Nurse-staffing levels and the quality of care in hospitals. New England Journal of Medicine, 346, 1715-1722. https://doi. org/10.1056/NEJMsa012247

Paris, M., \& Hoge, M. A. (2010). Burnout in the mental health workforce: a review. Journal of Behavioral Health Services \& Research, 37, 519-528. https://doi.org/10.1007/s11414-009-9202-2

Reineck, C., \& Furino, A. (2005). Nursing career fulfillment: statistics and statements from registered nurses. Nursing Economics, 23, 25-31.

Reschke, K. (2011). Medizinpsychologische Gesundheitsanalyse von Fahrerlehrer/innen in Bayern 2011 [Medical-psychological health analysis of driving instructors in Bavaria 2011]. Leipzig University.

Ruotsalainen, J., Verbeek, J., Marine, A., \& Serra, C. (2015). Preventing occupational stress in healthcare workers. Cochrane Database of Systematic Reviews, 4, CD002892. https://doi.org/10.1002/14651858.CD 002892.pub5

Saedpanah, D., Salehi, S., \& Moghaddam, L. F. (2016). Effect of emotion regulation training on occupational stress of critical care nurses. Journal of Clinical and Diagnostic Research, 10, 1-4. https://doi. org/10.7860/JCDR/2016/23693.9042

Sarid, O., Rivka, B., Rivka, E., \& Dorit, S. E. (2012). Are cognitive-behavioral interventions effective in reducing occupational stress among nurses? Applied Nursing Research, 25, 152-157. https://doi. org/10.1016/j.apnr.2011.01.004

Schröder, H., \& Reschke, K. (2010). Optimistisch den Stress meistern [Optimistic stress management]. Dgvt Verlag.

Smith, S. A. (2014). Mindfulness-based stress reduction: an intervention to enhance the effectiveness of nurses' coping with work-related stress. International Journal of Nursing Knowledge, 25, 119-130. https://doi.org/10.1111/2047-3095.12025

Watson J. (1986). A step in the right direction: Relaxation training for psychiatric staff. Senior Nurse, 5, 12-13.

Zeller, J. M., \& Levin, P. F. (2013). Mindfulness interventions to reduce stress among nursing personnel. Workplace Health and Safety, 61, 85-89. https:// doi.org/10.1177/216507991306100207 